\title{
Hypertriglyceridaemia - REDUCE-IT with icosapent ethyl
}

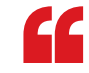

The clinical

benefits

exceeded what

I would expect

from the $20 \%$

reduction in

triglyceride

levels

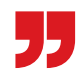

Use of icosapent ethyl is associated with a reduction in triglyceride levels and ischaemic events, including cardiovascular death, according to the results of the REDUCE-IT trial presented at the AHA Scientific Sessions. "The broad population of patients with elevated triglyceride [levels] we studied makes me believe that these results are widely applicable to millions of patients around the world," comments lead investigator, Deepak Bhatt.

Many individuals with cardiovascular risk factors and who are receiving appropriate statin therapy for primary or secondary prevention still have a substantial residual risk of having a cardiovascular event. In these individuals, elevated triglyceride levels are an independent marker of an increased risk of ischaemic events. However, current triglyceridelowering medications, such as niacin and fibrates, do not reduce the rates of cardiovascular events when given in addition to appropriate medical therapy, such as statins.

In the JELIS trial, eicosapentaenoic acid was shown to lower the risk of major coronary events in a Japanese population with hypertriglyceridaemia. The REDUCE-IT trial was designed

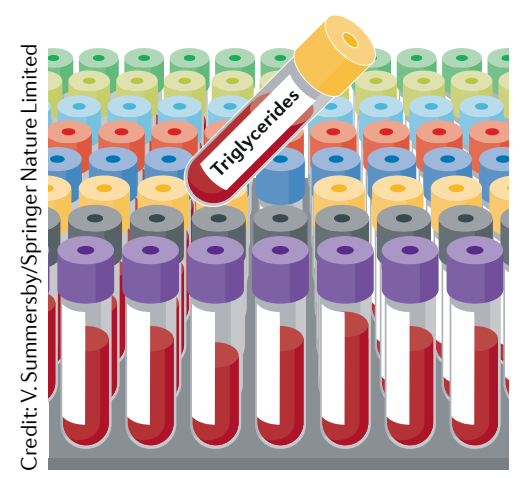

to test the effect of icosapent ethyl - a highly purified form of eicosapentaenoic acid - on cardiovascular outcomes. The trial included a total of 8,179 patients with established cardiovascular disease or diabetes mellitus and other risk factors who had been receiving statin therapy. Initial enrolment included patients who had a fasting triglyceride level of $135-499 \mathrm{mg} / \mathrm{dl}$ and an LDL-cholesterol level of 41-100 mg/dl, but as the study progressed, the minimum triglyceride threshold was increased to $200 \mathrm{mg} / \mathrm{dl}$. The baseline median levels of triglycerides and LDLcholesterol were $216.0 \mathrm{mg} / \mathrm{dl}$ and $75.0 \mathrm{mg} / \mathrm{dl}$, respectively. "The triglyceride entry criteria are important because they are a signal of potential residual risk that is not being managed by statin treatment," comments Robert Hegele, who was not involved in the study. "There is no approved therapy for the residual risk in these patients."

Participants were randomly assigned to receive $2 \mathrm{~g}$ of icosapent ethyl twice daily or placebo. The median change in triglyceride levels from baseline to 1 year was a decrease of $39.0 \mathrm{mg} / \mathrm{dl}$ in the icosapent ethyl group and an increase of $4.5 \mathrm{mg} / \mathrm{dl}$ in the placebo group. During follow-up (median 4.9 years), the rate of the primary end point (a composite of cardiovascular death, nonfatal myocardial infarction, nonfatal stroke, coronary revascularization or unstable angina) was $17.2 \%$ in the icosapent ethyl group and $22.0 \%$ in the placebo group (HR $0.75,95 \% \mathrm{CI}$ $0.68-0.83, P<0.001)$. The rate of the key secondary end point (a composite of cardiovascular death, nonfatal myocardial infarction or nonfatal stroke) was $11.2 \%$ and $14.8 \%$ in each group, respectively (HR 0.74, 95\% CI $0.65-0.83, P<0.001)$. The benefits were consistent across the range of baseline triglyceride levels. "I find the clinical effect sizes to be remarkable," says Hegele. "The clinical benefits exceeded what I would expect from the $20 \%$ reduction in triglyceride levels. This suggests other mechanisms are possibly contributing to the benefit," he adds.

Hegele suggests that potential pleiotropic effects of icosapent ethyl include an anti-inflammatory effect, given that the level of $\mathrm{C}$-reactive protein measured by high-sensitivity assay was lower with icosapent ethyl than with placebo. Also, a slightly higher risk of bleeding with active treatment indicates a possible anticoagulant mechanism. Furthermore, reductions in the rates of cardiac arrest and cardiac death with icosapent ethyl suggest a mechanism involving cell membrane stabilization.

Icosapent ethyl therapy was associated with an increased risk of atrial fibrillation and a trend towards an increased rate of serious bleeding, but Hegele believes "these small risks were offset by the overall much larger benefits in primary outcomes".

Icosapent ethyl is already used clinically for patients with triglyceride levels $\geq 500 \mathrm{mg} / \mathrm{dl}$ to decrease the risk of pancreatitis. "Potentially, when we look back, this line of therapy may be viewed as the dawn of a new era in cardiovascular [disease] prevention, much as statins were in years past," remarks Bhatt.

Gregory B. Lim

ORIGINAL ARTICLE Bhatt, D. L. et al. Cardiovascular risk reduction with icosapent ethyl for hypertriglyceridemia. N. Engl.J. Med. https://doi.org/10.1056/NEJMoa1812792 (2018) FURTHER READING Reiner, Ž. Hypertriglyceridaemia and risk of coronary artery disease. Nat. Rev. Cardiol. 14, 401-411 (2017) 